\title{
Dynamics of Layering Transitions in Confined Liquids
}

\author{
F. Mugele* and M. Salmeron ${ }^{\dagger}$ \\ Materials Sciences Division, Lawrence Berkeley National Laboratory, University of California, Berkeley, California 94720
}

(Received 1 November 1999)

\begin{abstract}
Multiple beam interferometry and video microscopy were used to investigate the layering transition of thin liquid films of 1-undecanol confined between atomically smooth mica surfaces. The expulsion of a molecularly thin lubricant layer was followed directly in two dimensions. Overall, the dynamics of the transition follows theoretical predictions based on two-dimensional hydrodynamics. Frequently, pockets of liquid remain trapped inside the contact area at the end of the transition. The trapped pockets undergo shape transformations to minimize elastic and interfacial energy.
\end{abstract}

PACS numbers: $68.15 .+\mathrm{e}, 61.20 .-\mathrm{p}, 68.45 .-\mathrm{v}$

The isotropy of a liquid is broken in the vicinity of an interface. As a result, its density oscillates as a function of distance from the surface with a periodicity roughly corresponding to one molecular diameter $h_{0}$ and with a decay length of a few diameters, provided that the interface is smooth on a molecular scale. This behavior has been established for a variety of systems including films of rare gases [1], liquid metals [2], and organic liquids [3-8]. For liquids in a narrow gap between smooth surfaces, the density oscillations originating on both surfaces overlap. This leads to oscillatory solvation forces when the surfaces are pressed together, accompanied by a series of layering transitions [3], each corresponding to a stepwise decrease of film thickness by the expulsion of a discrete amount of liquid $[4,5]$. Layering transitions have a significant bearing on the lubrication properties of thin films. For example, dissipative forces were found to vary strongly [6,7], and in some cases dramatically [8], when individual layers are expelled. However, there are no reports on the evolution of the process in two dimensions, except for the theoretical work of Persson and Tosatti [9].

Here we use multiple beam interferometry and video microscopy to investigate the layering transition of 1-undecanol films $\left(\mathrm{C}_{11} \mathrm{OH}\right)$ confined between atomically smooth back-silvered mica surfaces. The silver mirrors form a Fabry-Pérot interferometer. By choosing an appropriate filter, we select a narrow wavelength band $(\Delta \lambda=20 \mathrm{~nm})$ from a white light source to illuminate the sample, such that a significant fraction of the light is transmitted when the mica surfaces are brought into contact. When the surface separation is varied, the transmission peak of the interferometer (width $\approx 10 \mathrm{~nm}$ ) shifts. This leads to strong variations of the transmitted intensity, which are recorded using a CCD video camera with a microscope lens. The mica surfaces are mounted in crossed cylinder geometry inside a surface forces apparatus (SFA) that was described previously $[10,11]$. Normal forces are generated by magnetic fields produced by coils acting on a permanent magnet attached to a spring-support (spring constant $\approx 200 \mathrm{~N} / \mathrm{m}$ ) holding the lower mica sample. By abruptly switching on the coil current, we could change the normal force by several orders of magnitude within a short time $(<100 \mathrm{~ms})$. Immediately prior to the experiment, the mica surfaces were cleaved in a dry nitrogen atmosphere, to produce clean and very thin mica sheets $(d<1 \mu \mathrm{m})$ [12]. $\mathrm{C}_{11} \mathrm{OH}$ from Fluka (purity $>99.5 \%$ ) was used as received. The experiments were performed at room temperature $\left(22 \pm 1{ }^{\circ} \mathrm{C}\right)$.

As we have shown recently [13], the alcohol molecules form strongly bound monolayers on each mica surface that cannot be removed with pressures typically accessible in SFA experiments $(<100 \mathrm{MPa})$. Thus the $\mathrm{CH}_{3}$-terminated monolayers (the $\mathrm{OH}$ groups point towards the substrate) can be considered part of the substrate. The structure of the additional liquid is more complicated and depends on chain length and temperature $[14,15]$. For undecanol at room temperature, the molecules are arranged either in bilayers of highly tilted dimers $\left(>70^{\circ}\right)$ or in a more disordered layer with almost flat-lying molecules. The situation is not unlike that of confined liquid crystal films recently studied by Monte Carlo simulations [16]. Since the precise arrangement of the molecules is unknown under the nonequilibrium conditions of the present experiment, we will simply refer to a "layer" of alcohol molecules.

The experiments were performed as follows. Starting at a separation of several micrometers, the normal force is abruptly increased from 0 to $20 \mathrm{mN}$. As a result, the surfaces approach each other with a speed $>100 \mu \mathrm{m} / \mathrm{s}$ and most of the liquid between them is expelled. From experiments at low speed, we know that this load corresponds to a configuration in which the substrate-bound monolayers are in direct contact [13]. In the current experiment, however, a residual amount of liquid remains trapped in a metastable state due to the rapid approach. Within $<0.2 \mathrm{~s}$, the apexes of the two curved surfaces (initial radius of curvature $\approx$ $20 \mathrm{~mm}$ ) flatten out. The contact region [17] has an average radius $R=\left(r_{1} r_{2}\right)^{1 / 2}=40 \mu \mathrm{m}\left(r_{1}\right.$ and $r_{2}$ being the axes of the slightly elliptical contact area), corresponding to an average pressure of $4.3 \mathrm{MPa}$. Figure 1 shows the transmitted light intensity averaged over the contact area as a function of time. Within the first $4 \mathrm{~s}$ (phase I), the intensity decreases as liquid drains out of the gap in a process governed by three-dimensional hydrodynamics $[18,19]$. In phase II, the thickness remains constant, as shown by the 


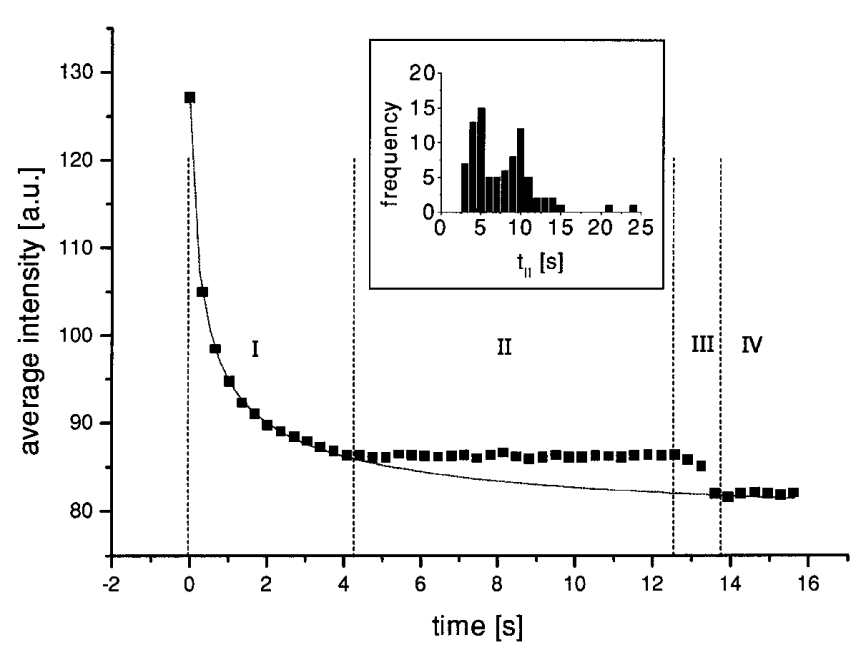

FIG. 1. Average transmitted light intensity through the contact area as a function of time during expulsion of the last unbound layer of $\mathrm{C}_{11} \mathrm{OH}$. The film thickness is a weakly nonlinear function of the intensity. Four phases (I to IV) characterize the layering transition. The inset shows the distribution of the duration of phase II $\left(t_{\mathrm{II}}\right)$ for a series of 85 expulsion cycles.

unchanging transmitted light intensity. After a time $t_{\mathrm{II}}$, the intensity drops by a discrete amount within a time interval $t_{\text {III }}<1 \mathrm{~s}$ (phase III). In the final state (phase IV), the film thickness remains constant indefinitely, corresponding to the two substrate-bound monolayers in contact. We conclude that the transition in phase III corresponds to the expulsion of the last unbound layer of molecules. After separating the surfaces by $>10 \mu \mathrm{m}$ and a waiting period of $5 \mathrm{~s}$, the cycle is repeated. Except for a variation of $t_{\mathrm{II}}$ (inset of Fig. 1), the same evolution was observed in a series of 85 cycles.

The dynamics of the layering transition during phase III can be followed by video images of the contact area, as shown in Fig. 2. The initial and final state images correspond to phases II and IV, respectively, averaged over several seconds. Figures 2(a)-2(f) are nonaveraged (hence noisier) video frames recorded in phase III. For clarity, we show difference images with the initial state image. Figure 2(a) shows the condition just prior to the expulsion. In Fig. 2(b), a darker area (encircled) becomes visible. We will refer to this area of reduced film thickness as a "hole island." As it grows, it quickly reaches the edge of the contact region. Then the boundary moves faster along the edge than in the central part, as seen in Figs. 2(c) and 2(d). The higher speed $(\approx 100 \mu \mathrm{m} / \mathrm{s})$ along the edge leads to negatively curved sections of the boundary. Some of these eventually detach, leaving behind pockets of trapped material. Note also that the boundary becomes rough on a shorter length scale. Generally the pockets are indefinitely stable [20]. The images in Fig. 2 represent a typical evolution of the boundary. Throughout the whole series of expulsion cycles, we observed on average three trapped pockets at random locations in the late stage of the transition.

The layering transition is separated into an initial phase (II), in which the nucleation takes place, and a subsequent growth phase (III). In the Persson and Tosatti (PT) model [9], nucleation is governed by the energy of a hole island:

$$
U(r)=2 \pi r \Gamma+\pi r^{2} \rho_{0}-\alpha r^{3} .
$$

The first term represents the line energy along the circumference of the hole island of radius $r$, with $\Gamma$ being the line tension. The second term is the change in the interface free energy, with $\rho_{0}$ being the spreading pressure. The third term represents the gain in elastic energy as the mica surfaces relax into the hole. Equation (1) predicts a critical nucleus size and energy, $U\left(r_{c}\right)$. The nucleation rate is then given by $\omega=\omega_{0} \exp \left[-U\left(r_{c}\right) / k_{B} T\right]$, with typical values of $\omega_{0} \approx 10^{17} \mathrm{~s}^{-1}$ and $r_{c} \approx 15 \AA$. If the film is in a quasiequilibrium state at the beginning of phase II, i.e., in a state with a spectrum of density fluctuations corresponding to the applied pressure and the momentary separation, an exponentially decaying probability of observing

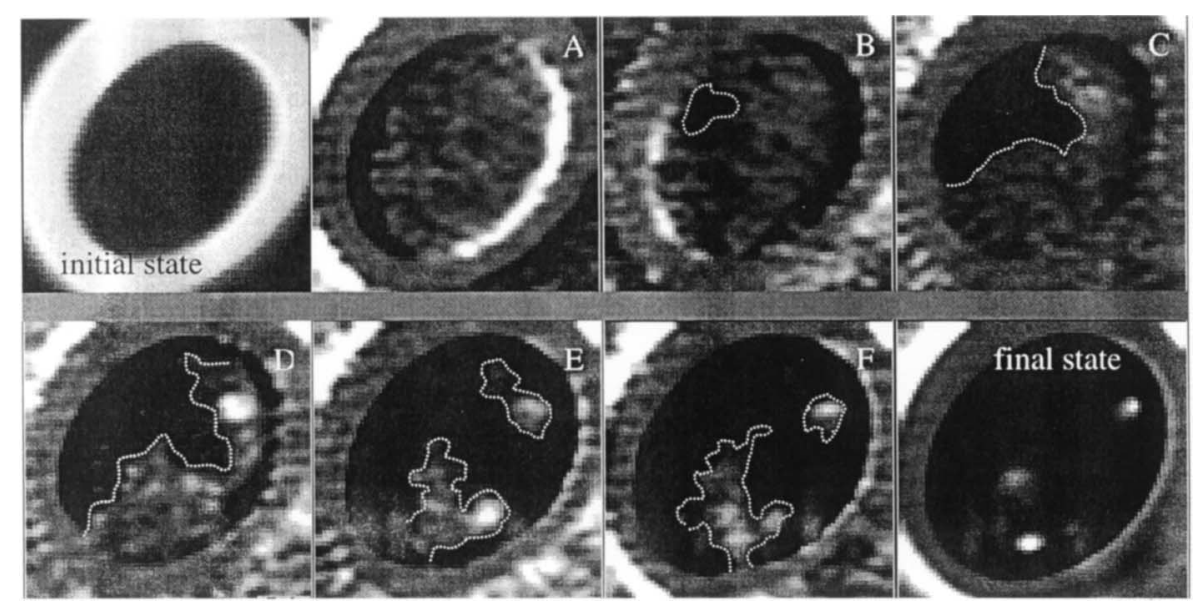

FIG. 2. Snapshots of the contact area during the layering transition (100 $\mu \mathrm{m} \times 100 \mu \mathrm{m}$ size). Images (a) to (f) are recorded at $0.16 \mathrm{~s}$ time intervals. Darker color corresponds to smaller film thickness. The dark and bright rims at the edge of the contact area in (b) and (c) are artifacts related to the video imaging. 
a layering transition per unit time would be expected. In contrast, the distribution of $t_{\mathrm{II}}$ displays two features (inset of Fig. 1). The first is that the layering transition never occurred within the first two seconds. Such a time lag is well-known in nucleation theory and is referred to as "induction" time when systems are quenched into metastable states by a rapid change of a thermodynamic variable [21]. In the present case, it is likely that the density of the layer is slightly enhanced after the rapid compression. Although no changes in the film thickness can be detected throughout phase II, a few percent of the molecules may diffuse out of the contact before a sufficiently large density fluctuation can build up. A similar two-stage process was observed in recent molecular dynamics simulations [22], in which a structural phase transformation that lowers the density is a prerequisite to hole nucleation. The second feature in the distribution of $t_{\mathrm{II}}$ is the presence of two peaks. We attribute this to changes in the nature of the defect acting as a nucleation site during data acquisition [23].

The liquid is expelled from the contact area with velocities of up to $100 \mu \mathrm{m} / \mathrm{s}$. The expulsion time $t_{\text {III }} \approx 1 \mathrm{~s}$ is 2 orders of magnitude smaller than the $\approx 120$ s found in experiments with spherical OMCTS molecules by Gee et al. [6]. Since the average pressure and contact area were similar to our experiment, the longer expulsion time must be related to significantly stronger friction forces opposing the process. In those measurements, a lateral shear force was applied to induce (and to monitor) the layering transition at a given load. Solidlike stick slip was observed both before and after the transition. In contrast, in our experiments with $\mathrm{C}_{11} \mathrm{OH}$, we found that the frictional force is very small before the transition and that stick-slip motion occurs only after the last layering transition. Therefore, the last-to-be-expelled $\mathrm{C}_{11} \mathrm{OH}$ layer must be in a liquidlike state. This is the physical explanation for the shorter expulsion time despite the more complex geometry of the molecules.

A liquidlike state is also a prerequisite for the growth model of PT, which is based on the two-dimensional Navier-Stokes equation. The latter is extended by inclusion of a drag force term $-\eta v$ ( $\eta$ is the friction coefficient and $v$ is the velocity), which describes energy dissipation when the layer slides across the substrate $[9,24]$. For the simple case of a circular nucleus in the center of the contact, $\eta$ can be calculated from the measured time $t_{\text {III }} \approx 1 \mathrm{~s}$. In this geometry, which should approximate the case of off-center nucleation to within a factor of $\approx 2$ [9], we obtain

$$
\eta=\frac{4 t_{\mathrm{III}} P_{\mathrm{ext}} h_{0}}{m n_{2 \mathrm{D}} R^{2}} \approx 10^{13} \mathrm{~s}^{-1} .
$$

Here, $m$ is the molecular mass of $\mathrm{C}_{11} \mathrm{OH}$, $n_{2 \mathrm{D}} \approx 1 /\left(30 \AA^{2}\right)$ the two-dimensional density, and $h_{0} \approx 8 \AA$ the thickness of the expelled layer. Uncertainties in the values of $n_{2 \mathrm{D}}$ and $h_{0}$ may add another factor of 2 to the error in $\eta$. PT applied the model to the data of Gee et al., in spite of the solidlike friction force, and obtained a value of $6 \times 10^{15} \mathrm{~s}^{-1}$ [25]. Most of the difference reflects the difference in the time $\left(t_{\text {III }}\right)$ of the transition in the two experiments.

At the late stage when the velocity depends only weakly on time, PT describe the hole-island's growth by a potential flow. Except for the roughness of the boundary and the trapped pockets, the predictions for the evolution of the boundary for off-center nucleation (Fig. 7 in Ref. [9]) agree strikingly well with our experimental result in Figs. 2(b)-2(d). The physics behind this behavior is as follows. The moving boundary has to push the molecules between its current position and the edge of the contact area along the direction of the flow lines. This gives rise to a resistance that is proportional to the length of this line. Therefore, the sections of the boundary closer to the edge of the contact move faster. The same mechanism explains the roughness of the boundary and the formation of trapped pockets. This effect should be observable within the PT model when a suitable perturbation of the initial boundary is assumed. Trapped pockets were indeed observed in recent simulations of a layering transition for a simple liquid [22].

We now analyze the stability of the trapped pockets. While some of them show the same transmitted light intensity as in the initial image, others are brighter. Figure 3 shows a sequence of images in which an initially flat pocket (top image; thickness $h_{0}$ ) is trapped and transforms into a brighter, i.e., thicker, one of reduced

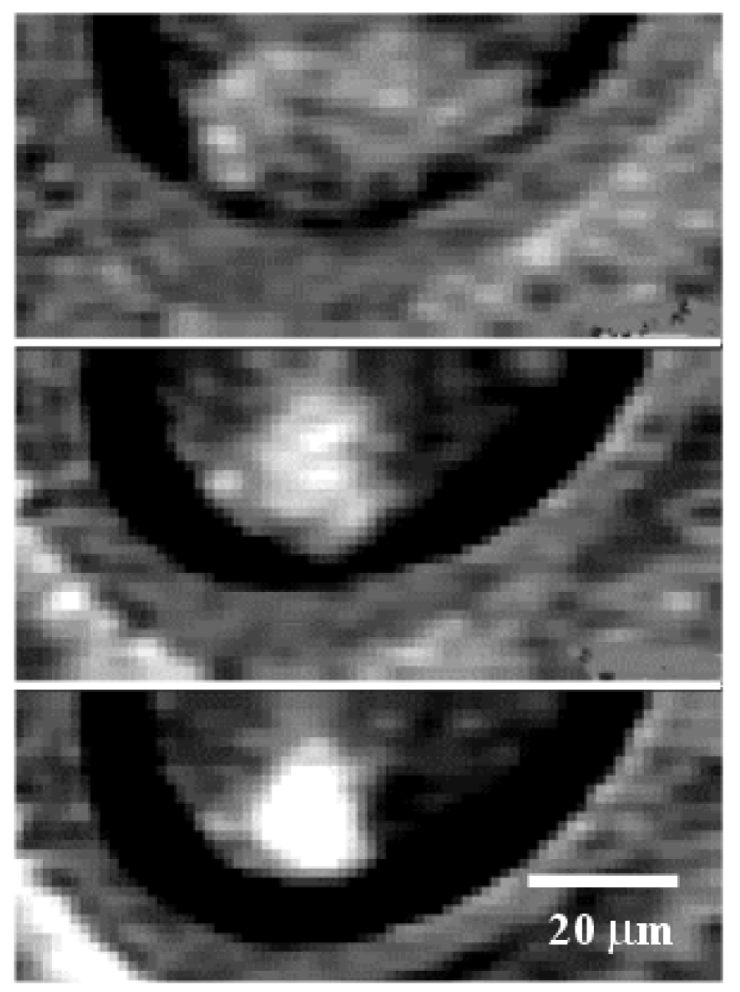

FIG. 3. Transformation of an initially flat pocket of trapped liquid into a thicker pocket of reduced diameter. Time difference between images is $0.16 \mathrm{~s}$. 
radius $r$. During this process, the interfacial area $\pi r^{2}$ and interfacial energy $\gamma \pi r^{2}$ are reduced, while the elastic energy due to the deformation of the mica increases. If we approximate the elastic energy by the work needed to press a rigid circular disk of radius $r$ and height $h$ by $h / 2$ into the semi-infinite elastic substrates on each side [26], we find

$$
U(r, h)=2 \gamma \pi r^{2}+\frac{E}{2\left(1-\nu^{2}\right)} r h^{2},
$$

where $E=1.75 \times 10^{10} \mathrm{~N} / \mathrm{m}^{2}$ is the elastic modulus and $\nu=0.44$ the Poisson ratio of mica. When $U(r, h)$ is minimized under the constraint of constant volume, we find that the optimal thickness gets larger as more liquid is trapped. To obtain a numerical estimate, we use $\gamma \approx 2 \mathrm{~mJ} / \mathrm{m}^{2}$ for the interfacial energy between the $\mathrm{CH}_{3}$-terminated substrate-bound molecules and bulk $\mathrm{C}_{11} \mathrm{OH}$ [27]. If we use the value of $8 \AA$ for $h_{0}$ [13], we find that one-layer-thick pockets will be stable for radii $r_{\mathrm{i}}$ of the initially trapped pocket up to $2 \mu \mathrm{m}$. For radii $r_{\mathrm{i}}$ of $2<r_{\mathrm{i}}<8 \mu \mathrm{m}$, $8<r_{\mathrm{i}}<19 \mu \mathrm{m}$, and $19<r_{\mathrm{i}}<35 \mu \mathrm{m}$, thickness values of $2 h_{0}, 3 h_{0}$, and $4 h_{0}$, respectively, are stable. Although we cannot resolve if the thickness of the pockets is discrete, the numerical values are reasonably close to the experimental results, suggesting that Eq. (3) correctly describes the physical processes.

In summary, the present results constitute the first direct investigation of a layering transition in two dimensions. The collapse of a lubricant layer after a rapid approach of the confining surfaces is divided into three phases. Initially, a molecularly thin lubrication layer is trapped in a metastable state. After an induction time, a layering transition is initiated by nucleation of a hole. The growth of the hole follows the predictions of two-dimensional hydrodynamics. Under the conditions of our experiment, the layering transition for $\mathrm{C}_{11} \mathrm{OH}$ films is incomplete, with a fraction of the lubricant layer trapped inside the contact area in the final state. Real-time mapping of molecularly thin liquid films can provide fundamental insights into the stability and dynamics of lubricant layers.

We thank B. N. J. Persson for stimulating and clarifying comments. F. M. acknowledges financial support by the Alexander von Humboldt Foundation in Germany. This work was supported by the Director, Office of Science, Office of Basic Energy Sciences, Materials Sciences Division of the U.S. Department of Energy under Contract No. DE-AC03-76SF00098.

*Present address: Abteilung Angewandte Physik, Universitaet Ulm, Albert Einstein Allee 11, D-89069 Ulm, Germany.

Electronic address: frieder.mugele@physik.uni-ulm.de
${ }^{\dagger}$ To whom correspondence should be addressed. Electronic address: salmeron@stm.lbl.gov

[1] D. S. Greywall and P. A. Busch, Phys. Rev. Lett. 67, 3535 (1991).

[2] O. M. Magnussen, B. M. Ocko, M. J. Regan, K. Penanen, P. S. Pershan, and M. Deutsch, Phys. Rev. Lett. 74, 4444 (1995).

[3] J. N. Israelachvili, Intermolecular and Surface Forces (Academic Press, New York, 1992), 2nd ed.

[4] J. Gao, W. D. Luedtke, and U. Landman, Phys. Rev. Lett. 79, 705 (1997).

[5] J. Gao, W. D. Luedtke, and U. Landman, J. Phys. Chem. B 101, 4013 (1997).

[6] M.L. Gee, P. M. McGuiggan, and J. N. Israelachvili, J. Chem. Phys. 93, 1895 (1990).

[7] A. L. Demirel and S. Granick, Phys. Rev. Lett. 77, 2261 (1996).

[8] J. Klein and E. Kumacheva, Science 269, 816 (1995).

[9] B. N. J. Persson and E. Tosatti, Phys. Rev. B 50, 5590 (1994).

[10] P. Frantz, A. Artsyukhovich, R. W. Carpick, and M. Salmeron, Langmuir 13, 5957 (1997).

[11] A. Artsyukhovich, L.D. Broekman, and M. Salmeron, Langmuir 15, 2217 (1999).

[12] P. Frantz and M. Salmeron, Tribol. Lett. 5, 151 (1998).

[13] F. Mugele, S. Baldelli, G. A. Somorjai, and M. Salmeron, J. Phys. Chem. B 104, 3140 (2000).

[14] S. J. O'Shea, M.E. Welland, and T. Rayment, Appl. Phys. Lett. 61, 2240 (1992).

[15] T. Nakada, S. Miyashita, G. Sazaki, H. Komatsu, and A. A. Chernov, Jpn. J. Appl. Phys. 35, L52 (1996).

[16] M. Schoen, T. Gruhn, and D. J. Diestler, J. Phys. Chem. 109, 301 (1998), and references therein.

[17] Initially the surfaces even bend inward due to elastohydrodynamic deformation. This transient effect disappears after $\approx 0.5 \mathrm{~s}$.

[18] D. Y. C. Chan and R. G. Horn, J. Chem. Phys. 83, 5311 (1985).

[19] J. N. Israelachvili, J. Colloid Interface Sci. 110, 263 (1986).

[20] Some pockets initially located close to the edge of the contact area do eventually disappear. They move closer to the edge and form little necks, such as the one at the bottom of Fig. 2(f), through which the liquid is squeezed out.

[21] See, for example, A. G. Walton, in Nucleation, edited by A. C. Zettlemoyer (Marcel Dekker, Inc., New York, 1969).

[22] B. N. J. Persson and P. Ballone (private communication); (to be published).

[23] These variations prevented a systematic study of the influence of quench speed on the layering transition.

[24] B. N. J. Persson, Sliding Friction (Springer-Verlag, Berlin, 1998).

[25] This is justified to the extent that the film undergoes repetitive melt-freeze cycles during stick-slip motion, as frequently assumed. In this case, $\eta$ represents an averaged friction coefficient.

[26] S.P. Timoshenko and J. N. Goodier, Theory of Elasticity (McGraw-Hill, New York, 1987), Chap. 12, p. 138.

[27] The correct value is probably smaller for a single layer of $\mathrm{C}_{11} \mathrm{OH}$, but on the same order of magnitude. 